\title{
Psychosocial Stress Among Patients With Type 2 Diabetes: Habitual Physical Activity as a Promising Moderator
}

\author{
Adeniyi, A.F., ${ }^{1}$ Ogwumike, O.O., ${ }^{1}$ Kolawole, E.B., ${ }^{1}$ Fasanmade, A.A. ${ }^{2}$ \\ ${ }^{1}$ Department of Physiotherapy, College of Medicine, University of Ibadan, Ibadan, Nigeria \\ ${ }^{2}$ Department of Medicine, University College Hospital, Ibadan, Nigeria \\ Correspondence \\ Dr A.F. Adeniyi, Department of Physiotherapy, College of Medicine, University of Ibadan, Ibadan, Nigeria \\ •E-mail: adeniyifatai@yahoo.co.uk; fadeniyi@comui.edu.ng
}

\begin{abstract}
SUMMARY
Psychosocial stress is a disabling condition and is common among people with diabetes mellitus in view of the complexity of the disorder. It is however not clear if the psychosocial stress has any link with habitual physical activity, which is an important component in the care of people with diabetes. This study was conducted to investigate the likely connection between habitual physical activity and psychosocial stress among people with type 2 diabetes. A total of 193 adults with type 2 diabetes took part in this study. Psychosocial stress was assessed using the Revised Questionnaire on Stress in patients with Diabetes (QSD-R) while physical activity was assessed using the Baecke Habitual Physical Activity Questionnaire. Data were analysed using descriptive and inferential statistics at $p<.05$. The mean age of the participants was $58.76 \pm 14.27$ years with mean duration of diagnosis being $6.41 \pm 4.51$ years. With a mean score of 2.8 , work activity was the main source of physical activity for the patients with the least activity index of 1.2 coming from sports participation. Hypoglycaemia and self-medication/diet were the most important sources of stress to the patients and the overall psychosocial stress was related to habitual physical activity $(r=-.73, p=.002)$. Significantly, patients with higher physical activity index presented with lower psychosocial stress. Apart from the well-known improvement in glycaemic control, type 2 diabetes patients that participate more in physical activity may be able to reduce their psychosocial stress.
\end{abstract}

KEY WORDS: Type 2 diabetes, psychosocial stress, habitual physical activity

\section{INTRODUCTION}

Stress is an inevitable part of life but it may directly or indirectly affect health behaviours. It is widely recognized that stress may have negative effects on health, and patients with type 2 diabetes may be at increased risk (Surwit et al., 2002). In a study by Herpertz et al. (2000), extreme psychosocial stress was found among $16.6 \%$ of patients with diabetes and those with extreme psychosocial stress showed worse diabetic control compared to less stressed patients. Psychosocial stress-related variables had also been reported as being significantly correlated with diabetes related factors such as hyperinsulinaemia, hyperglycaemia, dyslipidaemia, hypertension, increased abdominal obesity, and increased plasminogen activator inhibitor-1 (PAI-1) antigen (Raikkonen et al., 1996).

Schnohr et al. (2005) reported that due to increased physical activity in leisure time, joggers had a lower level of stress compared to sedentary persons. Hence it was recommended that increased well-being should be a key argument in future campaigns for increased leisure-time physical activity (Schnohr et al., 2005). With the complexities surrounding diabetes mellitus, the inverse relationship found between physical activity and psychosocial stress among the general population may not 
necessarily be assumed for patients with diabetes. Hence this study was conducted to investigate the connection that exists between psychosocial stress and habitual physical activity among patients with type 2 diabetes.

\section{METHODOLOGY}

\section{Participants}

A total of 193 adult volunteer participants took part in this cross-sectional study. They were type 2 diabetes patients being managed at either the Diabetes clinic of the University College Hospital (UCH) or the State Hospital, Ring Road, both in Ibadan as at the time of study. Participants for the study were recruited consecutively from both clinics. To be eligible to participate in the study, a participant had to be a type 2 diabetes patient who could read and/or write in English and/or Yoruba language, the main Nigerian language spoken in the region where the study was conducted. Patients with possible complications of diabetes that could affect participation in physical activity like amputation and blindness were excluded.

\section{Materials}

\section{Baecke Habitual Physical Activity Questionnaire}

The Baecke Habitual Physical Activity Questionnaire was administered on the participants to assess their physical activity. The questionnaire is a 12-item self-administered generic questionnaire which is used to objectively assess habitual physical activity of individuals. It was developed and first used in the Netherlands to investigate self-reported physical activity by Baecke, Burema and Frijters (Baecke et al., 1982). The questionnaire comprises 16 questions that investigate habitual physical activity over the past 12 months. It is divided into three sections: work (eight questions), sports/exercise (four questions), and non-sport leisure activities (four questions). The work activity section assesses activity level at work based on occupation, frequency of sitting, standing, walking, lifting, sweating, etc.; the sports activity section assesses activity level based on frequency of sweating while engaging in sports, leisure time sporting activity, duration of sporting activity etc; while the leisure activity section assesses activity level during leisure activities based on duration of watching television, walking, cycling, etc.

The Baecke Habitual Physical Activity Questionnaire employs a 5-point ordinal scale in assessing responses to the questions ranging from "never" to "sometimes" to "always". Scoring is as indicated by Baecke et al. (1982). Each category of habitual physical activity is scored and graded as follows:

Work activity is graded as:

Work index $=[(6-($ points for sitting $))+$ SUM (points for the other 7 parameters)] $/ 8$.

Sport activity is graded as:

Sport index $=[($ SUM (points for all 4 parameters $)] / 4$ Leisure activity is graded as:

Leisure index $=[(6-($ points for television watching $))$

+ SUM (points for remaining 3 items)] $/ 4$.

The mean index score was determined by the summation of the three sections divided by three:

(SUM (value for work index) + (value for sports index) + (value for leisure index) /3 (Kahan et al., 2005).

The questionnaire was tested for reliability and validity and test-retest validity was determined to be around 0.87 for Spearman's Rho (Rei et al., 2007). The validity and reliability of the Baecke Habitual Physical Activity Questionnaire has been found to range from fair to moderate in several studies. It has been frequently used in many studies as a general measure of physical activity (Misigoj-Durakovic et al., 2000; Eyler et al., 1997; Simons-Morton et al., 2000; Leslie et al., 2001; Livingstone et al., 2001) and was found to be highly reliable and valid for both men and women (Baecke et al., 1982). In a study by Hertogh et al. (2008) to examine the validity of the questionnaire, the score was assessed among 21 elderly men and women using the doubly labelled water method as the reference criterion. The study found the validity of the questionnaire to be fair-to-moderate with the Spearman correlation coefficient between the questionnaire score and the physical activity ratio as 0.54 (95\% CI $0.22-0.66)$.

\section{Questionnaire on Stress in Patients with Diabetes- Revised (QSD-R)}

The Questionnaire on Stress in patients with DiabetesRevised (QSD-R) is a self-assessment questionnaire for type I and type II diabetics, the purpose of which is to assess psychosocial stress associated with problems of daily living 
in people with diabetes and record the problems involved in coping with the illness and the treatment for it (Duran et al., 1995). The questionnaire was designed on the basis of interviews with patients, consultations with psychotherapists and reports from diabetes specialists. After conducting a comprehensive survey with the 90-item QSD, it was modified for clinical reasons (Duran et al., 1995). The revised QSD (QSD-R) contains 45 questions that define eight stress scales for patients with diabetes: leisure time, depression/fear of future, hypoglycaemia, treatment regimen/diet, physical complaints, work, partner, and doctor-patient relationship. The revised measure was assessed in 1,930 patients with both type 1 and type 2 diabetes. Cronbach's alpha ranged from 0.69 to 0.81 for the eight scales. Test-retest reliability for the eight scales ranged from 0.45 to 0.73 . Every statement in this questionnaire describes a possible impairment of everyday life, which must be assessed according to whether it applies to the test person (does not apply - applies) and (if it applies) the extent to which it is a problem (on a 5-point scale of $1=$ only a slight problem to $5=$ a very big problem). The higher the scores, the higher the level of stress felt by the respondent (Duran et al., 1995).

\section{Procedure}

Ethical approval of the University of Ibadan/University College Hospital Ethics Review Committee was sought and obtained before commencing the study. An informed consent form stating the purpose of the study as well as assuring participants of confidentiality and anonymity was attached to the questionnaire to be administered. The questionnaires were translated into Yoruba following standard procedures for the benefit of Yoruba speaking participants who do not understand or speak English. The questionnaires were self-administered, but assistance was available for those who needed help. The questionnaires were administered at the respective outpatient diabetic clinics of the selected hospitals. Socio-demographic information including age, gender, and occupation, level of education, marital status and time/period since diagnosis, was obtained from the subjects.

\section{Data Analysis}

Descriptive statistics of mean and standard deviation were used to analyse all continuous data. Frequencies and percentages were used to summarize all categorical data.
Spearman's correlation was used to evaluate the association between habitual physical activity and stress scores. Data was analysed using IBM SPSS Statistics 20.0 (IBM Corp., Armonk, NY, USA). Level of significance was set at $\mathrm{p}=$ 0.05 .

\section{RESULTS}

About two-thirds (68.3\%) of the participants were females with the mean of the participants' age being $58.76 \pm 14.27$ years. More than half of the participants $(52.21 \%)$ had been diagnosed with type 2 diabetes for over 10 years and the majority of them $(83.60 \%)$ were married.

The physical activity index of the participants (figure 1) shows that work activity was the main source from which the participants derived their physical activity. The work physical activity index of 2.8 was about double that of sports (1.2) and leisure activities (1.4). The QSD-R has 8 domains and each item has 6 response options from "does not apply" to "a very big problem". The results of the most prevalent response by the participants concerning each of the domains are as presented in table 1 . The table shows that "a very big problem" was the most reported response for the domains of hypoglycaemia and self-medication/diet. This implies that these two domains were the most stressful. All the other domains also reflected varying levels of stress with the least reported in leisure time. There was a significant inverse relationship between habitual physical activity and stress scores $(r=-0.73 ; p=0.002)$ (table 2$)$.

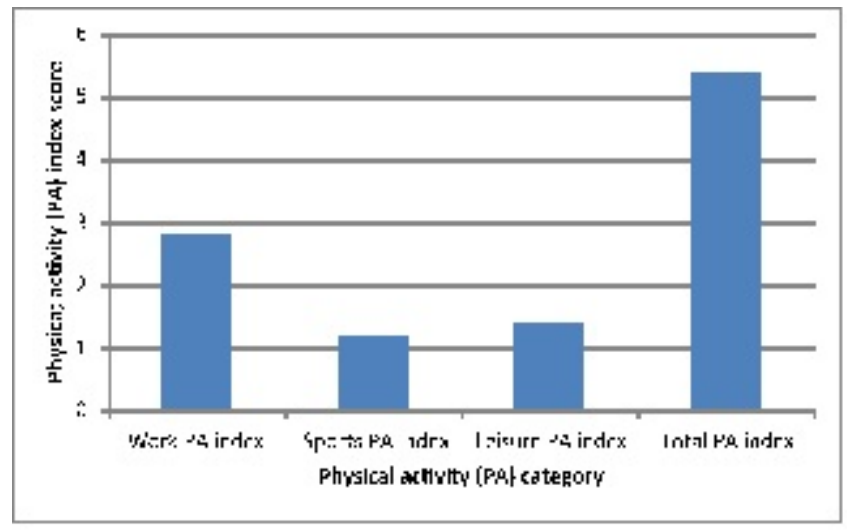

Figure 1. Physical activity distribution of the participants Key: $\mathrm{PA}=$ Physical activity 
Table 1. Distribution of participants' psychosocial stress according to frequency of response

\begin{tabular}{|c|c|c|c|c|c|c|}
\hline \multirow[b]{2}{*}{ Stress domains } & \multicolumn{6}{|c|}{ Most frequent score in the domain } \\
\hline & $\begin{array}{c}\begin{array}{c}\text { Does not } \\
\text { apply }\end{array} \\
0\end{array}$ & $\begin{array}{c}\text { Only a slight } \\
\text { problem } \\
1\end{array}$ & 2 & 3 & 4 & $\begin{array}{c}\text { A very big } \\
\text { problem } \\
5\end{array}$ \\
\hline \multicolumn{7}{|l|}{ Leisure time (items $2,9,18,30$ ) } \\
\hline \multicolumn{7}{|c|}{ Depression/fear of future (items $28,34,40,42,43,45$ ) } \\
\hline \multicolumn{7}{|l|}{ Hypoglycaemia (Items 12, 22, 25, 31) } \\
\hline \multicolumn{7}{|c|}{ Self-medication/diet (items $1,5,8,10,13,21,26,37,39$ ) } \\
\hline \multicolumn{7}{|c|}{ Physical complaints (items $6,17,19,20,38,44$ ) } \\
\hline \multicolumn{7}{|l|}{ Work (items $4,11,16,27,33,41$ ) } \\
\hline \multicolumn{7}{|l|}{ Partner (items $3,14,15,24,35,36$ ) } \\
\hline Doctor-patient relationship (items 7, 23, 29, 32) & & & & & & \\
\hline
\end{tabular}

Table 2. Relationship between habitual physical activity and psychosocial stress

\begin{tabular}{lll}
\hline & r & p value \\
\hline Total PA index score & & \\
Vs & -0.73 & 0.002 \\
Stress Scores & & \\
\hline
\end{tabular}

\section{DISCUSSION}

Findings from this study show that the main physical activity performed by the patients was in the line of their daily occupational activities. The patients performance was lowest in the area of sports activities. This finding points to the fact that the particular set of patients with type 2 diabetes that were involved in this study were only engaged in activities that had to do with their respective jobs and had less activity outside of their jobs. This trend was previously reported by the Centers for Disease Control and Prevention (2000) which observed that approximately half of adults who reported no physical activity during leisure time interestingly reported that they performed at least 1 hour per day of hard physical activity at work. This trend is not appropriate for the total well-being of the patients. In addition, a study by Sisson et al. (2009) reported that in men, high Leisure Time Sedentary Behaviour (LTSB) is associated with higher odds of metabolic syndrome and individual cardiovascular disease risk factors regardless of meeting physical activity recommendations. In women, high LTSB is associated with higher odds of metabolic syndrome only in those not meeting the physical activity recommendations. It was reported that both leisure and occupational physical activities are important components of a healthy lifestyle for people with diabetes ( $\mathrm{Hu}$ et al., 2004).

The participants provided responses for all the 8 domains of the QSD-R, but the most frequent report of serious concern about psychosocial stress was in the hypoglycaemia and self-medication/diet domains. Hypoglycaemia has consistently been a problem for people with diabetes. According to a previous study, profiles of psychosocial stress showed maximal stress with regard to depression in both types of diabetes; and this was followed by fear of hypoglycaemia in the sub-sample of type 1 and physical complaints in the sub-sample of type 2 diabetics (Herpertz et al., 2000). It was also reported that selfreported hypoglycaemia was prevalent among individuals with type 2 diabetes and it is associated with lower healthrelated quality of life, and a higher level of depression (Lustman and Clouse, 2005; Green et al., 2012).

The inability of patients with diabetes to consume foods of their choice is also a major contributor to psychosocial stress. It has been known that patients with type 2 diabetes have a number of issues that are related to their disorder and are capable of introducing psychosocial stress into their daily life. In describing everyday problems involved in living with diabetes, it was reported that dietary restrictions, self-monitoring of blood glucose, taking insulin injections, and lack of support from family and health care professionals (Rubin and Peyrot, 2001) are commonplace. The current study also found that issues like work, partner and physician relationships were important factors in the psychosocial stress pathway of the participants in this study. 
An inverse but significant relationship was discovered between habitual physical activity and psychosocial stress scores. This implies that a high level of psychosocial stress could be linked to low physical activity, although this could not be causally linked. The relationship found between habitual physical activity and psychosocial stress scores could be explained from a variety of dimensions. Considering the fear of physical activity reported by patients with diabetes (Thomas et al., 2004; Brazeau et al., 2008; Lascar et al., 2014), it means that the fear of having hypoglycaemia is likely to be stressful to the patients and by extension have a downward effect on their participation in physical activity.

However, an interesting aspect of the inverse relationship between habitual physical activity and psychosocial stress is the fact that it is not known which of the two variables influenced the other. For example, a type 2 diabetic patient who is inactive physically is likely to present with problems bordering on the domain areas of the QSD-R. Typically, a patient who is not sufficiently active may present with stress because of poor glycaemic control, and such a patient will need to make up for the inactivity through increased medication, and a strict diet, which may further contribute to the stress observed in the patient. Conversely, a patient who is psychosocially stressed is not likely to engage in sufficient physical activity and may have poor diabetic control (Herpertz et al., 2000). This is because in addition, stress can disrupt diabetes control indirectly through effects on diet, exercise, and other selfcare behaviours (Surwit et al., 2002). This situation was also reported in a meta-analysis conducted by Chida and Hamer (2008).

The clinical relevance of this study lies in its ability to inform changes in the educational strategy used for patients with type 2 diabetes. It is commonplace for the patients to complain of psychosocial stress while simultaneously neglecting an important component of their care which is regular physical activity. A considerable number of diabetic patients suffer from extreme psychosocial stress often associated with poor diabetic control (Herpertz et al. 2000). Hence it may be instructive to educate patients about the fact that physical activity may help reduce their level of psychosocial stress because it serves as an important moderator to some of the various components of their health that play together to generate psychosocial stress. It is however important to note that the relationship observed in this study cannot be said to be causal hence caution has to be exercised in interpreting the results. In addition, the socio-demographic factors of the participants may also have influenced the relationship.

\section{CONCLUSION AND RECOMMENDATION}

It is concluded that a significant link was found between habitual physical activity and psychosocial stress among patients with type 2 diabetes. Patients with a high level physical activity presented with lower psychosocial stress. Beyond glycaemic control, patients with type 2 diabetes may have improved psychosocial health if they engage in sufficient physical activity.

\section{References}

Baecke J.A., Burema J. and Frijters J.E. 1982. A short questionnaire for the measurement of habitual physical activity in epidemiological studies. American Journal of Clinical Nutrition 36: 936-842.

Brazeau A.S., Rabasa-Lhoret R., Strychar I. and Mircescu H. 2008. Barriers to physical activity among patients with type 1 diabetes. Diabetes Care 31(11): 2108-2109.

Centers for Disease Control and Prevention (CDC). 2000. Prevalence of leisure-time and occupational physical activity among employed adults - United States, 1990. Morbidity and Mortality Weekly Report 49(19): 420-424.

Chida Y. and Hamer M. 2008. An association of adverse psychosocial factors with diabetes mellitus: A meta-analytic review of longitudinal cohort studies. Diabetologia 51(12): 2168-2178.

Duran G., Herschbach P., Waadt S., Strain F. and Zettler A. 1995. Assessing daily problems with diabetes: A subject-oriented approach to compliance. Psychological Reports 76: 515-521.

Eyler A.A., Borwnson R.C. and King A.C. 1997. Physical activity and women in the United States: an overview of health benefits, prevalence, and intervention opportunities. Women Health 26: 27-249.

Green A.J., Fox K,M. and Grandy S. for the SHIELD Study Group. 2012. Self-reported hypoglycemia and impact on quality of life and depression among adults with type 2 diabetes mellitus. Diabetes Research and Clinical Practice 96(3): 313-318.

Herpertz S., Johann B., Lichtblau K., Stadtbäumer M., Kocnar M., Krämer-Paust R. et al. 2000. Patients with diabetes mellitus: Psychosocial stress and use of psychosocial support: a multicenter study. MedizinischeKlinik 95(7): 369-377.

Hertogh E.M., Monninkhof E.M., Schouten E.G., Peeters H.M. and Schuit A.J. 2008. Validity of the Modified Baecke Questionnaire: comparison with energy expenditure according 
to the doubly labeled water method. International Journal of Behavioral Nutrition and Physical Activity 5: 30.

doi:10.1186/1479-5868-5-30

Hu G., Eriksson J., Barengo N.C., Lakka T.A., Valle T.T., Nissinen A., Jousilahti P. and Tuomilehto J. 2004. Occupational, commuting, and leisure-time physical activity in relation to total and cardiovascular mortality among Finnish subjects with type 2 diabetes. Circulation 110: 666-673.

Kahan E., Fogelman Y. and Bloch B. 2005. Correlations of work, leisure, and sports physical activities and health status with socioeconomic factors: a national study in Israel. Postgraduate Medical Journal 81: 262-265. doi:10.1136/ pgmj.2004.022293

Lascar N., Kennedy A., Hancock B., Jenkins D., Andrews RC., Greenfield S. and Narendran P. 2014. Attitudes and barriers to exercise in adults with type 1 diabetes (T1DM) and how best to address them: A qualitative study. PLOS ONE 9(9): e108019. doi:10.1371/journal.pone.0108019

Leslie E., Fortheringham M.J., and Owen N. 2001. Age-related differences in physical activity levels of young adults. Medical Science in Sports Exercise 33: 255-258.

Livingstone M.B., Robson P.J., and McCarthy S. 2001. Physical activity patterns in a nationally representative sample of adults in Ireland. Public Health Nutrition 4: 1107-1116.

Lustman P.J. and Clouse R.E. 2005. Depression in diabetic patients: The relationship between mood and glycemic control. Journal of Diabetes and its Complications 19(2): 113-122.

Misigoj-Durakovic M., Heimer S., and Matkovic B.R. Physical activity of urban adult population: questionnaire study. Croatian Medical Journal 2000; 41: 428-432.

Räikkönen K., Keltikangas-Järvinen L., Adlercreutz H. and
Hautanen A. 1996. Psychosocial stress and the insulin resistance syndrome. Metabolism 45(12): 1533-1538.

Rei O, Hirata S., Yamada M., Nishiyama T., Kurosaka M. and Tamura Y. 2007. Reliability and validity of the Baecke physical activity questionnaire in adult women with hip disorders. Biomedical Central 8: 61.

Rubin R.R. and Peyrot M. 2001. Psychological issues and treatments for people with diabetes. Journal of Clinical Psychology 57(4): 457-478.

Schnohr P., Kristensen T.S., Prescott E. and Scharling H. 2005. Stress and life dissatisfaction are inversely associated with jogging and other types of physical activity in leisure time-The Copenhagen City Heart Study. Scandinavian Journal of Medicine \& Science in Sports 15(2): 107-112.

Simons-Morton D.G., Hogan P, and Dunn A.L. 2000. Characteristics of inactive primary care patients: baseline data from the activity counseling trial. Preventive Medicine 31: 513-321.

Sisson S.B., Camhi S.M., Church T.S., Martin C.K., Tudor-Locke C., Bouchard C. et al. 2009. Leisure time sedentary behavior, occupational/domestic physical activity, and metabolic syndrome in U.S. men and women. Metabolic Syndrome and Related Disorders 7(6): 529-536.

Surwit R.S., Van Tilburg M.A.I., Zucker N., Mccaskill C.C., Parekh P., Feinglos M.N. et al. 2002. Stress management improves long-term glycemic control in type 2 diabetes. Diabetes Care 25: 30-34.

Thomas N., Alder E. and Leese, G.P. 2004. Barriers to physical activity in patients with diabetes. Postgraduate Medical Journal 80(943): 287-291. 